\title{
ISOLAMENTO SOCIAL PELA PANDEMIA COVID-I9 NO MANEJO DA DOR CRÓNICA
}

\author{
AISLAMIENTO SOCIAL POR LA PANDEMIA COVID-I9 EN EL MANEJO DEL \\ DOLOR CRÓNICO
}

\section{SOCIAL ISOLATION BY THE COVID-i9 PANDEMIC IN THE MANAGEMENT OF THE CHRONIC PAIN- SCOPING REVIEW}

\author{
Inês Pereira ${ }^{1}$ \\ João Mourinho ${ }^{2}$ \\ Joana Galvão ${ }^{3}$ \\ Marta Rodrigues ${ }^{4}$ \\ Paulo Sabino $^{5}$ \\ Rafaela Santos ${ }^{6}$ \\ Alice Ruivo ${ }^{7}$
}

\begin{abstract}
RESUMO: Objetivos: Mapear a evidência científica acerca da influência do isolamento social por Covid-ı9 no Manejo da Dor Crónica. Sugerir ações que minimizem as interferências da Pandemia por Covid-ı9 no manejo da dor crónica. Método: Foi seguida a metodologia JBI (Joanna Briggs Institute), a pesquisa foi realizada entre outubro e novembro de 2020 nas bases de dados Biblioteca do Conhecimento, Ebscohost, PubMed Central (PMC) e PubMed, foi considerado o espaço temporal de janeiro de 2019 a dezembro de 2020 e conjugados os boleanos "and" e "not". A Scoping Review (SR) pretende mapear os procedimentos metodológicos e partiu da seguinte questão central: "O manejo da dor crónica foi afetado pelo Isolamento Social causado pela Pandemia por Covid-ı??", sendo esta definida pela questão PCC. Resultados: Do universo de 862 artigos encontrados foram selecionados 16 após aplicação dos critérios de exclusão. Conclusão: Corroboramos que se revela fulcral adotar medidas individualizadas no acompanhamento do doente com dor crónica em fase de Pandemia por Covid-ı, de modo a diminuir as comorbilidades nestes doentes e aumentar a qualidade de vida.
\end{abstract}

Palavras-Chave: Isolamento Social. Manejo da Dor. Dor Crónica e Covid-ı9.

RESUMEN: Objetivos: Mapear la evidencia científica sobre la influencia del aislamiento social por Covid-ıg en el manejo del dolor crónico. Sugerir acciones que minimicen la interferencia de Pandemic por Covid-ı́ en el manejo del dolor crónico. Método: Se siguió la metodología JBI (Joanna Briggs Institute), la investigación realizada entre octubre y noviembre de 2020 en las bases de datos biblioteca del conocimiento, Ebscohost, PubMed Central (PMC) y PubMed, se consideró el espacio temporal de enero de 2019 a diciembre de 2020 y el combinado "y" y "no" booleanos. La Scoping Review (SR) tiene la intención de mapear los procedimientos metodológicos y partió de la siguiente pregunta central: “¿El manejo del dolor crónico fue afectado por la pandemia por Covid-ı9?”, Que se define en la pregunta del PCC. Resultados: Del universo de 862 artículos encontrados, se seleccionaron i6 después

${ }^{I} R N$, Enfermeira de Cuidados Gerais na NefroEstremoz - Estremoz, Portugal; ORCID: https://orcid.org/ooooooo2-9554-5753

${ }^{2}$ RN, Enfermeiro de Cuidados Gerais no Hospital do Espírito Santo EPE, - Évora, Portugal;

${ }^{3}$ RN, Enfermeira de Cuidados Gerais na Medicina II, Hospital José Joaquim Fernandes - ULSBA, EPE, Beja, Portugal. ORCID: https://orcid.org/oooo-ooo3-2288-3169

${ }^{4}$ RN, Enfermeira de Cuidados Gerais na Medicina ala Direita do Hospital de Santa Luzia - ULSNA, EPE, Elvas, Portugal. ORCID: https://orcid.org/oooo-0oo2-5026-oIr3

${ }^{5}$ RN, Enfermeiro de Cuidados Gerais na Medicina II Hospital José Joaquim Fernandes - ULSBA, EPE, Beja, Portugal. ORCID: https://orcid.org/oooo-ooo3-2329-3072

${ }^{6}$ RN, Enfermeira de Cuidados Gerais na Medicina II Hospital de Faro - CHUA, EPE, Faro, Portugal. ORCID: https://orcid.org/oooo-ooor-6995-7722

7 PhD, Professora Coordenadora da Escola Superior de Saúde do Instituto Politécnico de Setúbal; Membro do CIIAS, Mestre em Psicologia, Doutorada em Psicologia de Intervenção; Especialista em Enfermagem MédicoCirúrgica. ORCID: https://orcid.org/oooo-oooz-696o-828X 
de aplicar los critérios de exclusión. Conclusión: Corroboramos que es fundamental adoptar medidas individualizadas para el seguimiento del paciente con dolor crónico en fase pandémica por Covid-I9, con el fin de reducir las comorbilidades en estos pacientes y aumentar la calidad de vida.

Palabras clave: Aislamiento social. Manejo del dolor. Dolor crónico y Covid-ı.

ABSTRACT: Objectives: To map scientific evidence about the influence of social isolation by Covid-I9 on Chronic Pain Management. Suggest actions that minimize the interference of Covid-r9 Pandemic in the management of chronic pain. Method: The JBI (Joanna Briggs Institute) methodology was followed, the research was carried out between October and November 2020 in the Knowledge Library, Ebscohost, PubMed Central (PMC) and PubMed databases, the temporal space from January 2019 to December was considered 2020 and the combined "and" and "not" Booleans. The Scoping Review (SR) intends to map the methodological procedures and started from the following central question: "Was the management of chronic pain affected by the Social Isolation caused by the Pandemic by Covid-I9?", which is defined by the PCC question. Results: of the universe of 862 articles found, I6 were selected after applying the exclusion criteria. Conclusion: we corroborate that it is crucial to adopt individualized measures to monitor the patient with chronic pain in Pandemic phase by Covid-I9, in order to reduce the comorbidities in these patients and increase the quality of life.

Keywords: Social Isolation. Pain Management. Chronic Pain and Covid-19

\section{INTRODUÇÀO}

A dor é definida como "uma experiência sensitiva e emocional desagradável, associada, ou semelhante àquela associada, a uma lesão tecidual real ou potencial” ${ }^{(\mathrm{r})}$, deve ser valorizada para poder ser tratada. A mesma é reconhecida como quinto sinal vital desde janeiro de 200o, nos EUA, desde a publicação de uma norma pela JCAHO, enquanto em Portugal, é assim considerada desde $2003^{(2)}$, implementando a avaliação e registo sistemático da dor em todos os serviços prestadores de cuidados de saúde.

Para abordar a dor importa clarificar que a sua classificação, pode ser diferenciada quanto à fisiopatologia e à duração. Quanto à fisiopatologia, podemos referir a existência de dor nociceptiva, dor neuropática e dor psicossomática. Já quanto à duração, distingue-se entre dor aguda, uma "dor de início recente e de provável duração limitada" (3) e dor crónica, "quando, de forma contínua ou recorrente, existe há 3 meses ou mais, ou quando persiste para além do curso normal de uma doença aguda ou da cura da lesão que lhe deu origem"(4). A Dor Crónica é, muitas vezes, limitante e de difícil controlo, independentemente da patologia de base que a causa, tem implicações na saúde e na qualidade de vida dos doentes(5). Torna-se ainda relevante refletir acerca do conceito de dor total, exposto por Cicely Saunders em 
1967, o qual refere que a dor transpõe a dimensão física, abrangendo também aspetos psicológicos, sociais e espirituais do sofrimento ${ }^{(6)}$.

Com a atual pandemia por Covid-I9, as sociedades viram-se forçadas a adotar medidas no sentido de mitigar a disseminação de Covid-ı, tendo, para tal, adotado medidas de isolamento e distanciamento físico. Daí advieram limitações ao uso de serviços públicos, forçando as instituições de saúde a reorganizar e redistribuir os seus recursos, o que alterou o funcionamento dos serviços não urgentes, onde se incluem os serviços de controlo da dor. Desta forma, o eficaz controlo da dor tornase um desafio, existindo o risco de danos por tratamento inadequado. Pessoas com dor, frequentemente relatam aumento da intensidade da mesma quando se sentem socialmente isoladas( 7 ), até porque existem estudos que sugerem "que as relações sociais têm um papel importante na dor" ${ }^{(8)}$, acrescentando ainda que os indivíduos com maior suporte social revelam uma maior tolerância à dor ${ }^{(8)}$.

Verificando-se a pertinência da temática, elaborou-se uma SR cujo objetivo principal é identificar a interferência da pandemia por Covid-ı9, no manejo da dor crónica.

METODO: Foi elaborada uma SR, tendo a escolha recaído neste tipo de desenho de pesquisa devido ao facto de se verificar escassez de evidências consideradas fortes do ponto de vista metodológico na temática a abordar. As SR "são consideradas ferramentas válidas para mapear evidências disponíveis"(9) mas também para “esclarecer conceitos-chave e identificar lacunas de conhecimento"(9).

Para a elaboração desta SR, seguiu-se o modelo do Joanna Briggs Institute $(\mathrm{JBI})^{(\mathrm{ro})}$. A estrutura desta SR consiste em seis principais etapas consecutivas, sendo estas: I) Identificação da questão PCC e objetivo de pesquisa; 2) Identificação de estudos relevantes, que viabilizassem a amplitude e abrangência dos propósitos da revisão; 3) Seleção de estudos, documentos, conforme os critérios predefinidos; 4) Mapeamento de dados; 5) Sumarização dos resultados, por meio de uma análise temática qualitativa em relação ao objetivo e pergunta e por último; 6) Apresentação dos resultados, identificando as implicações para política, prática ou pesquisa.

Primeiramente, procedeu-se à formulação da questão PCC: O manejo da dor crónica foi afetado pelo Isolamento Social causado pela Pandemia por Covid-I9? Neste sentido, procurámos: conhecer os efeitos do Isolamento Social pela Pandemia 
por Covid-I9 no manejo da dor crónica e sugerir ações que minimizem as interferências da Pandemia por Covid-ı9 no manejo da dor crónica.

Considerámos como critérios de inclusão de artigos: participantes: Doente com dor crónica; contexto: Pandemia por Covid-ı;; conceito: Manejo da dor crónica em tempo de Pandemia por Covid-I9. Foram considerados todos os tipos de estudo (primários e secundários), que incluam manejo da dor crónica em tempo de pandemia Covid-I9, disponíveis em full- text e PDF, com autores da área da saúde e pesquisas efetuadas entre 2019-2020 em português, inglês ou espanhol. A pesquisa foi realizada no período de outubro a novembro de 2020 , tendo sido adotada uma estratégia com três etapas ${ }^{(\mathrm{Io})}$ :

$\mathrm{Na}$ primeira etapa, recorreu-se à pesquisa nas bases de dados $\mathrm{B}-\mathrm{ON}$, Ebscohost, PMC e PubMed, por serem abrangentes e terem ampla cobertura das publicações na área da saúde, o que permitiu definir os descritores a utilizar.

$\mathrm{Na}$ segunda etapa, foi realizada pesquisa nas referidas bases de dados, tendo sido aplicados os descritores DECS e MESH: "social isolation”, “pain management", "chronic pain" e "Covid-r9".

Assim, no domínio EBSCOHOST, foram selecionadas as bases de dados CINAHL PLUS with Full Text, Nursing and Allied Health Collection, Cohcrane Plus Collection, MedicLatina e Medline with Full Text, sendo posteriormente aplicados os descritores acima mencionados. Inicialmente, aplicaram-se todos os descritores, aplicando o operador boleano “AND”. Pelo fato de não terem surgido resultados, reformulou-se a forma de pesquisa e assim aplicaram-se os boleanos da seguinte forma [Social Isolation AND Pain Management OR Chronic Pain AND Covid-r9].

$\mathrm{Na}$ base de dados PubMed, a utilização de descritores e boleanos processou-se da forma acima descrita. Refinou-se a busca utilizando os filtros referidos, com a seleção das bases Medline e Nursing Journals e a aplicação do assunto selecionado Humans.

$\mathrm{Na}$ base de dados Biblioteca do Conhecimento, para além da aplicação de descritores, boleanos e filtros mencionados, aplicou-se ainda "Disponível na coleção da Biblioteca", Revistas Académicas" e "Revistas" e foram colocados como assuntos "chronic pain", "social isolation" e "Covid-ı"”.

$\mathrm{Na}$ base de dados PMC, refinou-se a pesquisa com o filtro Texto de acesso livre. 
Após aplicação dos critérios de inclusão, as razões mais comuns para a exclusão dos estudos foi por não abordarem a consequência do isolamento social no manejo da dor crónica em tempos de pandemia Covid-ı.

$\mathrm{Na}$ terceira etapa, procedeu-se à análise dos 16 artigos encontrados. A metodologia efetuada fica esquematizado no PRISMA $S_{c} R^{(\text {II) }}$ (figura I).
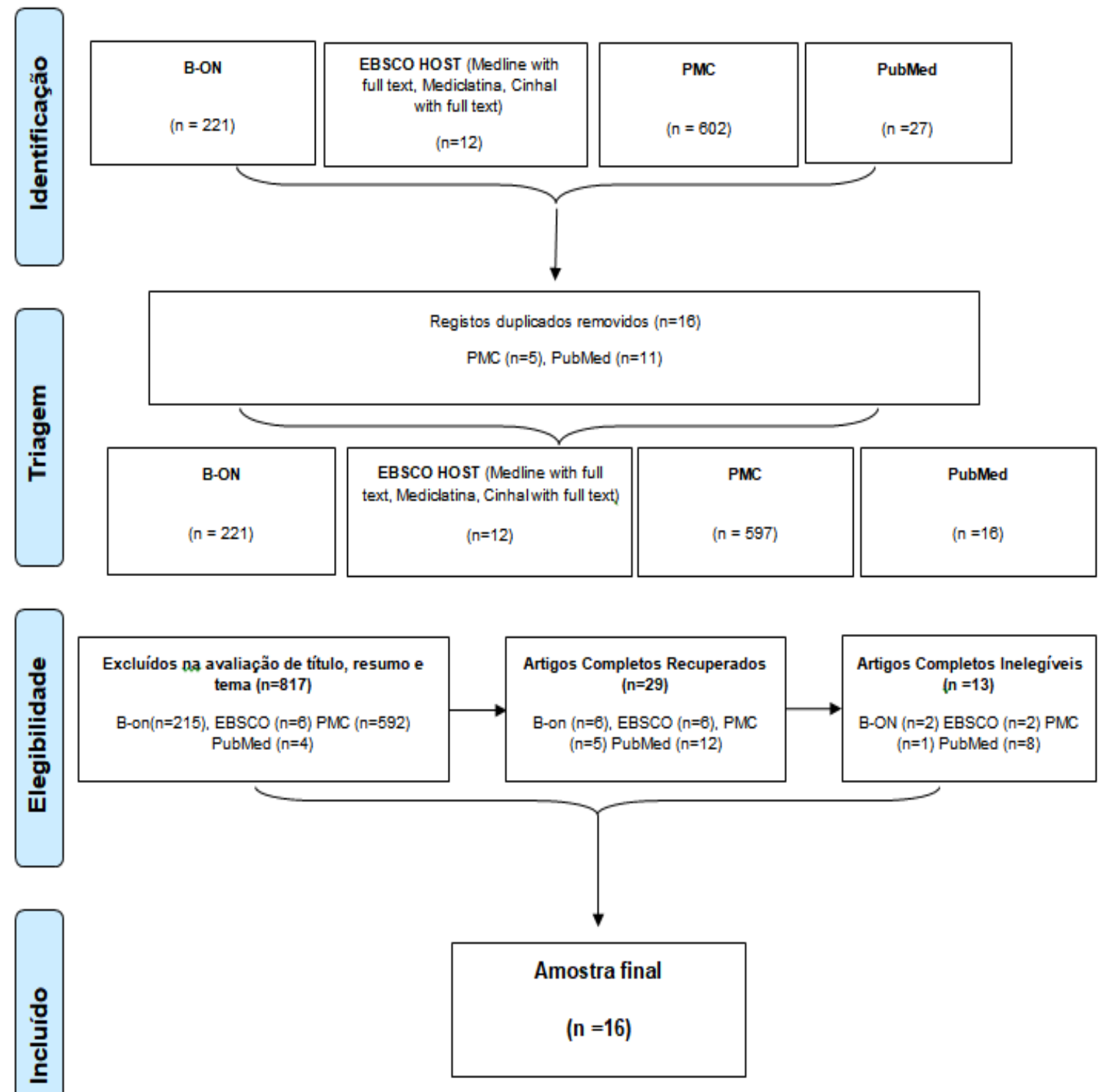

Figura I. Prisma Scoping Review Flow Chart ${ }^{(1}$

\section{RESULTADOS}

A Extração dos dados foi realizada por dois revisores de forma independente e, nos casos de conflito, recorreu-se a um terceiro, seguindo as orientações da JBI. 


\begin{tabular}{|c|c|c|c|c|}
\hline \multicolumn{5}{|c|}{ Extração de Dados- B-ON } \\
\hline ID & Objetivo & Participantes & Conceito & $\begin{array}{c}\text { País/ } \\
\text { Contexto }\end{array}$ \\
\hline 12 & $\begin{array}{l}\text { Informar acerca do } \\
\text { manejo da dor crónica } \\
\text { na era Covid-ı9 }\end{array}$ & Não aplicável & $\begin{array}{l}\text { Carta ao editor. Aborda as consequências das medidas de distanciamento e isolamento social, identificando- } \\
\text { os como fatores de exacerbação de sintomas depressivos e ideação suicida, já de si presentes em doentes com } \\
\text { dor crónica. Refere o cancelamento de serviços de controlo da dor imposto pela pandemia. Refletindo acerca } \\
\text { da manutenção destes serviços, para casos selecionados, considerados urgentes. É sugerida a telemedicina } \\
\text { como solução. }\end{array}$ & $\begin{array}{l}\text { (Italiana/ Irlanda } \\
\text { do Norte) } \\
\text { Pandemia Covid- } \\
\text { I9 }\end{array}$ \\
\hline 13 & $\begin{array}{l}\text { Analisar o impacto da } \\
\text { pandemia Covid-ı́ no } \\
\text { tratamento da dor } \\
\text { crónica e indicar } \\
\text { estratégias para } \\
\text { manter o tratamento. }\end{array}$ & $\begin{array}{l}\text { Referências que } \\
\text { abordam a } \\
\text { temática em } \\
\text { estudo }\end{array}$ & $\begin{array}{l}\text { Revisão que aborda o impacto da pandemia por Covid-ıg em doentes com dor crónica e respetivo manejo. } \\
\text { Referindo que a dor crónica está associada a fatores emocionais, afetivos e sociais, que são agravados } \\
\text { durante o isolamento, tornando os cuidados de saúde mais desafiadores, nesta fase em relação ao tratamento } \\
\text { da dor. Alude o risco de não tratar a dor ou de tratamento inadequados. Releva intervenções inadiáveis no } \\
\text { controlo da dor, havendo consultas que se têm que manter presenciais e outros casos menos complexos } \\
\text { devem ser tratados em consulta de telessaúde, controlando os sintomas dos doentes em casa. Refere como } \\
\text { limitação da telessaúde: questões de proteção de dados, doentes com défices cognitivos idosos ou com afeção } \\
\text { linguística. }\end{array}$ & $\begin{array}{l}\text { (Itália/ } \\
\text { PUA) } \\
\text { Pandemia } \\
\text { COVID-ı́ } \\
\text { nível global }\end{array}$ \\
\hline 14 & $\begin{array}{l}\text { Identificar o impacto } \\
\text { da pandemia Covid-ı9 } \\
\text { no manejo da dor } \\
\text { crónica e fornecer } \\
\text { sugestões }\end{array}$ & $\begin{array}{l}\text { Doentes com } \\
\text { dor crónica }\end{array}$ & 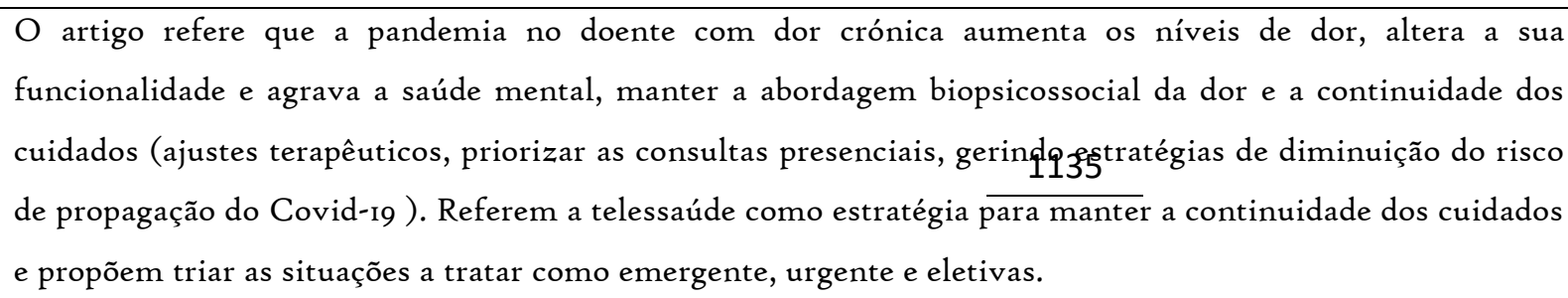 & $\begin{array}{l}\text { (Paquistão) } \\
\text { Pandemia } \\
\text { COVID-ı9 }\end{array}$ \\
\hline
\end{tabular}




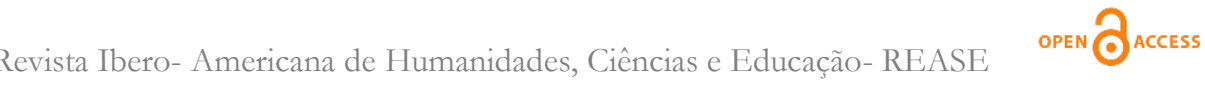

\begin{tabular}{|c|c|c|c|c|}
\hline 15 & $\begin{array}{l}\text { Descrever os desafios } \\
\text { dos doentes com dor } \\
\text { crónica na pandemia e } \\
\text { o uso da telessaúde } \\
\text { como forma de } \\
\text { continuidade ao } \\
\text { tratamento }\end{array}$ & $\begin{array}{l}\text { Doentes com } \\
\text { dor crónica }\end{array}$ & $\begin{array}{l}\text { O tratamento da dor crónica revela-se essencial, uma vez que este tipo de dor é a razão mais comum para } \\
\text { procurar ajuda médica. O contexto de Pandemia, levou ao encerramento de vários serviços (tratamento de } \\
\text { dor). Doentes com dor crónica têm maior risco de ansiedade, stress e depressão, sendo agravados em tempo } \\
\text { de pandemia, o ineficaz tratamento da dor leva a consequências para indivíduos, sociedade e sistema de } \\
\text { saúde a curto e longo prazo. A suspensão de consultas da dor interfere com os mecanismos que os doentes } \\
\text { com dor desenvolveram para fazer face à fadiga e à dor. A telessaúde está a emergir como solução para uma } \\
\text { comunicação eficiente de modo a fornecer serviços de saúde mantendo a continuidade dos cuidados, refere } \\
\text { como limitações, a falta de clarificação médico/legal. }\end{array}$ & $\begin{array}{l}\text { (Índia) } \\
\text { Pandemia Covid- } \\
\text { I9 na Índia }\end{array}$ \\
\hline
\end{tabular}

\begin{tabular}{|c|c|c|c|c|}
\hline \multicolumn{5}{|c|}{ Extração de Dados- PMC } \\
\hline ID & Objetivo & Participantes & Conceito & $\begin{array}{c}\text { País/ } \\
\text { Contexto }\end{array}$ \\
\hline 16 & $\begin{array}{l}\text { Compreender as } \\
\text { alterações no sistema } \\
\text { de saúde durante a } \\
\text { pandemia Covid-19 } \\
\text { Aconselhar os } \\
\text { profissionais de } \\
\text { saúde acerca da } \\
\text { gestão de doentes } \\
\text { com dor crónica. }\end{array}$ & $\begin{array}{l}\text { Integrados } \\
\text { estudos sobre a } \\
\text { temática }\end{array}$ & $\begin{array}{l}\text { Explora mudanças do sistema de saúde decorrentes do isolamento durante a pandemia, que influenciam o } \\
\text { tratamento da dor crónica. O Isolamento tem forte efeito nos doentes com dor, adiando ou suspendendo } \\
\text { os tratamentos, levando a consequências como: aumento da dor, depressão, aumento da ansiedade, maior } \\
\text { imobilidade, incapacidade e comportamentos aditivos (álcool e opioides). Apresenta soluções, como: } \\
\text { triagem meticulosa dos doentes, afirmam a telessaúde como solução, permitindo a gestão terapêutica } \\
\text { através de ferramentas audiovisuais, no manejo da dor. No entanto referem como limitação a abordagem } \\
\text { de casos inaugurais. Afirmam também a importância de uma triagem adequada e uma monitorização da } \\
\text { dor meticulosa, mesmo sendo realizada remotamente e releva a abordagem multidisciplinar e o } \\
\text { envolvimento do doente na tomada de decisão. }\end{array}$ & $\begin{array}{l}\text { (Arábia Saudita, } \\
\text { Egito e EUA) } \\
\text { Pandemia } \\
\text { COVID-19 }\end{array}$ \\
\hline 17 & $\begin{array}{l}\text { Formular } \\
\text { recomendações } \\
\text { práticas para dar } \\
\text { continuidade aos } \\
\text { cuidados no âmbito } \\
\text { da dor crónica }\end{array}$ & $\begin{array}{l}\text { Painel de } \\
\text { peritos na área } \\
\text { da dor crónica } \\
\text { (médicos e } \\
\text { psicólogos) }\end{array}$ & $\begin{array}{l}\text { Realizada uma pesquisa literária, os autores não encontraram documentos ou guideliness referentes ao } \\
\text { controlo da dor crónica em tempos de pandemia. Em resposta } 136 \text { esta lacuna, são formuladas } \\
\text { recomendações para guiar a prática no cuidado ao doente com dor crónica. Para tal, foi convocado um } \\
\text { painel de peritos em dor crónica. Durante a pandemia existe risco de doentes com dor crónica não } \\
\text { receberem os devidos tratamentos na sequência das medidas de contenção tomadas. No artigo identifica- } \\
\text { se a telessaúde como forma de manter a continuidade dos cuidados e evitar deslocaços desnecessárias ao } \\
\text { hospital, mantendo os cuidados biofísicosociais de forma remota e assegurando os ajustes terapêuticos, }\end{array}$ & $\begin{array}{l}\text { (Canadá, EUA, } \\
\text { Portugal, Holanda) } \\
\text { Pandemia Covid-19 }\end{array}$ \\
\hline
\end{tabular}




\begin{tabular}{|c|c|c|c|c|}
\hline & & & tendo por base a avaliação do doente e dos seus sintomas. & \\
\hline 18 & 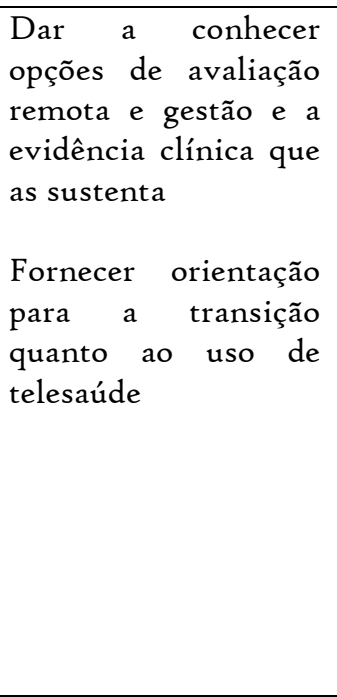 & $\begin{array}{l}\text { Incluídos } \\
\text { Estudos sobre a } \\
\text { temática a } \\
\text { abordar }\end{array}$ & $\begin{array}{l}\text { Em situação de pandemia, refere-se a necessidade de manter os tratamentos de forma remota, mais } \\
\text { especificamente na dor crónica, sendo considerados } 4 \text { fatores para orientar os profissionais no cuidar } \\
\text { destes doentes. I. consequências do Covid-ı́ para os doentes com dor crónica, 2. consequências de não } \\
\text { tratar a dor durante o período de pandemia, 3. Opções para a avaliação remota da dor, 4. Evidências } \\
\text { clínicas que suportem o tratamento remoto; São abordadas opções de tratamento à distância, como a } \\
\text { telessaúde e é sugerida a apresentação de soluções de melhoria destes modelos de trabalho. A aposta na } \\
\text { prevenção e tratamento da dor crónica é uma necessidade nos cuidados de saúde, quando é comprometido } \\
\text { o acesso ao tratamento de dor crónica há agravamento acentuado da dor. Níveis elevados de dor } \\
\text { dificultam a funcionalidade, aumentando a depressão, diminuindo a qualidade de vida. Apontam a } \\
\text { telessaúde (consultas telefónicas, sms, partilha de imagem, documentação e videoconferências), como } \\
\text { método preferencial de fazer frente ao distanciamento e relevam a necessidade de auto-gestão da dor, para } \\
\text { isso o doente é munido de competências e treino para o controlo da dor (através da sua avaliação e gestão } \\
\text { de medidas farmacológicas e não farmacológicas.) A telessaúde poderá ter limitações, na avaliação } \\
\text { principalmente quando há grande descontrolo sintomático ou quando a relação empática ainda não está } \\
\text { estabelecida, a maior inovação é o desenvolvimento de terapias para controlo da dor de forma remota. } \\
\text { Não houve diferença no controlo da dor entre o grupo com apoio remoto e o grupo com consultas } \\
\text { presenciais. Aponta limitações para o uso das tecnologias nos adultos/idosos e pessoas com deficiências. }\end{array}$ & $\begin{array}{l}\text { (Canadá e } \text { EUA) } \\
\text { Pandemia Covid-I9 }\end{array}$ \\
\hline 19 & $\begin{array}{l}\text { Dar a conhecer o } \\
\text { funcionamento da } \\
\text { assistência remota } \\
\text { em saúde, como } \\
\text { forma de assistência } \\
\text { a doentes com dor } \\
\text { crónica }\end{array}$ & Não aplicável & $\begin{array}{l}\text { Reflete as consequências do isolamento: ansiedade e depressão, que de si está aumentado em doente com } \\
\text { dor crónica. Os autores abordam as oportunidades para a aplicação das plataformas digitais, bem como as } \\
\text { suas limitações, a necessidade de serem avaliados os conteúdos e procedimentos por parte de profissionais } \\
\text { de saúde para garantir a sua qualidade, o suporte e continuidade dos cuidados através de: Plataformas de } \\
\text { telessaúde, Comunidades de saúde online, Inteligência artificial (chats com sistemas de resposta padrão) } \\
\text { Permitindo a recolha de dados, triagem. Sublinha a multidisciplinaridade inerente à abordagem da dor. } \\
\text { Apontando a telessaúde como ferramenta de modo a evitar a presença física do doente. }\end{array}$ & $\begin{array}{l}\text { (Singapura, } \\
\text { Londres) } \\
\text { Pandemia Covid-I9 }\end{array}$ \\
\hline
\end{tabular}

\begin{tabular}{|c|c|c|c|c|}
\hline \multicolumn{5}{|c|}{ Extração de Dados- PUBMED } \\
\hline ID & Objetivo & Participantes & Conceito & $\begin{array}{c}\text { País/ } \\
\text { Contexto }\end{array}$ \\
\hline 20 & $\begin{array}{l}\text { Descrever guidelines que } \\
\text { forneçam estrutura para } \\
\text { gerir riscos e permitir } \\
\text { acesso a serviços de }\end{array}$ & $\begin{array}{l}\text { Peritos na área de } \\
\text { manejo da dor } \\
\text { crónica (no ramo } \\
\text { das forças armadas) }\end{array}$ & $\begin{array}{l}\text { Decorreu uma conferência com peritos na área do manejo da dor crónica para estabelecimento de linhas } \\
\text { orientadoras para retomar em segurança intervenções de manejo da dor crónica de forma segura. } \\
\text { ASPETOS GERAIS DE MITIGAÇÃO DA INFEÇO (uso de máscara, promover a higiene das } \\
\text { mãos, avaliação da temperatura, divulgar medidas de segurança, triagem de doentes com febre e }\end{array}$ & $\begin{array}{l}\text { USA } \\
\text { Pandemia } \\
\text { Covid-r9 }\end{array}$ \\
\hline
\end{tabular}




\begin{tabular}{|c|c|c|c|c|}
\hline & manejo da dor & & $\begin{array}{l}\text { sintomas respiratórios, limitar os acompanhantes dos doentes, restruturar os serviços para manter a } \\
\text { distância de segurança), MEDIDAS ESPECÍfICAS (doentes suspeitos, infetados ou com contactos de } \\
\text { risco deve usar } \mathrm{FP}_{2} \text { ). }\end{array}$ & \\
\hline 21 & $\begin{array}{l}\text { Refletir sobre as } \\
\text { consequências da } \\
\text { pandemia Covid-ı em } \\
\text { termos de manejo de dor } \\
\text { crónica. }\end{array}$ & $\begin{array}{l}\text { Estudos que } \\
\text { abordam a temática } \\
\text { em estudo }\end{array}$ & $\begin{array}{l}\text { A dor crónica pode ser exacerbada por agravamento da dor física já existente e dos distúrbios mentais. } \\
\text { Pode haver novos diagnósticos de dor crónica ligados à Covid-ig e ao isolamento a que obriga. O } \\
\text { impacto da Covid-ıg far-se-á sentir em toda a população (os que foram infetados e os que tiveram de } \\
\text { mudar a sua rotina diária pelo isolamento), levando ao agravamento da dor crónica pelo adiamento de } \\
\text { consultas, maior inatividade e aumento de stressores psicológicos. }\end{array}$ & $\begin{array}{l}\text { (EUA, } \\
\text { Alemanha e } \\
\text { Canadá) } \\
\text { Pandemia } \\
\text { Covid-ı9 }\end{array}$ \\
\hline 22 & $\begin{array}{l}\text { Reflexão-abordagem aos } \\
\text { doentes com dor crónica } \\
\text { em tempos de pandemia } \\
\text { Covid-ı }\end{array}$ & Não aplicável & $\begin{array}{l}\text { Define controlo da dor e afirma que o Covid-ıg levou à suspensão de muitos serviços considerados não } \\
\text { urgentes, afirma a telessaúde como forma de controlar a dor em tempos de pandemia, sublinha a relação } \\
\text { entre dor e isolamento. Refere ainda a triagem meticulosa dos doentes com dor para avaliar os que } \\
\text { necessitam de consultas presenciais ou remota. }\end{array}$ & $\begin{array}{l}\text { (EUA) } \\
\text { Pandemia } \\
\text { Covid-I9 }\end{array}$ \\
\hline 23 & $\begin{array}{l}\text { Determina os efeitos do } \\
\text { isolamento na dor } \\
\text { crónica. }\end{array}$ & $\begin{array}{l}\text { Estudos sobre a } \\
\text { temática }\end{array}$ & $\begin{array}{l}\text { Revisão que aborda as consequências das medidas impostas pela pandemia no manejo da dor crónica. } \\
\text { Releva a importância de manter medidas de controlo da dor, preferencialmente de forma remota. }\end{array}$ & $\begin{array}{l}\text { Espanha } \\
\text { Pandemia } \\
\text { Covid-ı9 }\end{array}$ \\
\hline
\end{tabular}

\begin{tabular}{|c|c|c|c|c|}
\hline \multicolumn{5}{|c|}{ Extração de Dados- EBSCO } \\
\hline ID & Objetivo & Participantes & Conceito & $\begin{array}{c}\text { País/ } \\
\text { Contexto }\end{array}$ \\
\hline 24 & $\begin{array}{l}\text { Descrição de protocolo } \\
\text { para retomar } \\
\text { procedimentos de manejo } \\
\text { da dor crónica de forma } \\
\text { segura considerando a } \\
\text { pandemia. }\end{array}$ & $\begin{array}{c}\text { Médicos } \\
\text { especialistas } \\
\text { em manejo de } \\
\text { dor }\end{array}$ & $\begin{array}{l}\text { Artigo em que é proposto um protocolo (não testado) de reintegração de serviços, para reiniciar de forma } \\
\text { segura intervenções de manejo da dor em tempo de pandemia. Este modelo baseia-se em } 8 \text { passos, para } \\
\text { manter a segurança de doentes e profissionais de saúde: I. Agendamento (triagem e planeamento de } \\
\text { tratamentos) 2. Testagem para COVID-ıg (em caso de contacto presencial), restrições no atendimento } 3 . \\
\text { Distanciamento Social 4. Chegada dos doentes (distanciamento e número reduzido de doentes e } \\
\text { profissionais) 5. Àrea de Recuperação 6.Sala de Fluroscopia (sala higienizada) 7. Equipamento de Proteção } \\
\text { individual (viseira nos procedimentos, máscara FP2) 8. Informaçoês aquando da Alta Clínica (indicações, } \\
\text { agendamento de consulta de follow up preferencialmente remota.) }\end{array}$ & $\begin{array}{l}\text { EUA } \\
\text { Montefiore: } \\
\text { Programa } \\
\text { Multi } \\
\text { disciplinar de } \\
\text { Manejo da } \\
\text { Dor, durante } \\
\text { a pandemia } \\
\text { Covid-I9 }\end{array}$ \\
\hline
\end{tabular}




\begin{tabular}{|c|c|c|c|c|}
\hline 25 & $\begin{array}{l}\text { Fornecer informação e } \\
\text { diretrizes para retomar as } \\
\text { intervenções de controlo da } \\
\text { dor crónica, durante a } \\
\text { pandemia Covid-ı. } \\
\text { minimizando riscos } \\
\quad \text { associados. }\end{array}$ & $\begin{array}{c}\text { Médicos } \\
\text { especialistas } \\
\text { em manejo de } \\
\text { dor }\end{array}$ & $\begin{array}{l}\text { Descreve recomendações para retomar em segurança práticas de manejo da dor crónica, reforçando a } \\
\text { necessidade de haver EPI's e a telessaúde como forma de manter os cuidados. Foi criado um painel de } \\
\text { peritos no controlo da dor, que formulou recomendações baseadas na estratificação de doentes quanto a } \\
\text { fatores de risco. Negligenciar os cuidados a este doente tem repercussões negativas na sua qualidade de vida, } \\
\text { estabilidade psicológica e controlo de sintomas, abordaram formas coerentes de retomas atividade de forma } \\
\text { segura, tendo em conta o risco de mitigação. Refere também aumento da ansiedade, depressão, maior } \\
\text { imobilidade e comportamentos aditivos (álcool e opioides). }\end{array}$ & $\begin{array}{c}\text { EUA } \\
\text { Pandemia } \\
\text { Covid-I9 }\end{array}$ \\
\hline 26 & $\begin{array}{l}\text { Refletir o manejo da dor } \\
\text { crónica durante a } \\
\text { pandemia COVID-i9 }\end{array}$ & Não aplicável & $\begin{array}{l}\text { Reflexão, que aborda os desafios dos especialistas no manejo da dor crónica na pandemia. Utilizando a } \\
\text { metáfora de Homero da obra Odisseia, analisa a redistribuição dos recursos para enfrentar o vírus SARS- } \\
\text { COV } 2 \text { e as consequências para o manejo da dor crónica, que fica comprometido ao ser tido como } \\
\text { procedimento não urgente. Defendem que o tratamento da dor crónica deve ser considerado urgente, } \\
\text { correndo o mesmo risco de Homero, ao direcionar os recursos para o Covid-ı, relegando para segundo plano } \\
\text { todas as outras patologias. }\end{array}$ & $\begin{array}{c}\text { EUA } \\
\text { Pandemia } \\
\text { COVID-i9 }\end{array}$ \\
\hline 27 & $\begin{array}{l}\text { Fornecer orientação para } \\
\text { retomar intervenções no } \\
\text { âmbito do manejo da dor } \\
\text { crónica }\end{array}$ & $\begin{array}{c}\text { Médicos } \\
\text { especialistas } \\
\text { em manejo de } \\
\text { dor }\end{array}$ & $\begin{array}{l}\text { Aborda o impacto da Covid-ı na sociedade, em particular no controlo da dor crónica. A Covid-ıg fez } \\
\text { interromper o cuidado da dor e a vida como estávamos acostumados a vivê-la. Aponta a telessaúde como } \\
\text { solução, embora reconheça limitações (doentes "naif" para opióides que iniciaram tratamento) levada a cabo } \\
\text { pela American Society of Intervencional Pain Physicians (ASIIP) para orientar os profissionais na triagem } \\
\text { de intervenções (emergente, urgentes, eletivas) de manejo da dor crónica. Desenvolvendo uma Escala de } \\
\text { Acuidade de intervenções decidir tratamentos a efetuar, categorizando as situações como emergentes, } \\
\text { urgentes e eletivas. }\end{array}$ & $\begin{array}{c}\text { USA } \\
\text { Pandemia } \\
\text { Covid-ı9 }\end{array}$ \\
\hline
\end{tabular}




\section{DISCUSSÃO}

Com a presente SR, e com vista a dar resposta aos objetivos formulados, para discussão, os resultados foram agrupados em: consequências do isolamento social pela pandemia Covid-ı9 no manejo da dor crónica e soluções que visam a continuidade do manejo da dor crónica em tempos de pandemia Covid-ı.

- Consequências do Isolamento Social pela Pandemia COVID-r9 no Manejo da Dor Crónica.

Considerando as referências integradas nesta SR, de um modo geral, fica patente que o manejo da dor crónica fica comprometido em tempos de pandemia Covid-ig. Este facto prende-se com a necessidade de redistribuição de recursos humanos para fazer face a esta pandemia, bem como o encerramento de serviços considerados não urgentes, onde os serviços de controlo da dor se inserem ${ }^{(12,18,21,26)}$. Serviços direcionados para o tratamento da dor encerraram nesta época de pandemia, por todo o mundo, sendo que procedimentos não emergentes foram interrompidos ou cancelados $^{(14,17)}$. Esta situação colocou os cuidados aos doentes com dor crónica em risco. É importante considerar que a grande maioria destes indivíduos se caraterizam por serem idosos e com múltiplas co-morbilidades associadas e, portanto, integram o grupo de risco para contrair infeção por SARS-COV̌ e, como consequência, Covid$19^{(14)}$. Também as políticas adotadas para travar a pandemia, distância e isolamento social, são apontadas como fatores de risco para o agravamento da condição clínica dos doentes com dor crónica. Tem-se feito o apelo à população de evitar recorrer aos serviços de saúde de forma leviana, para mitigar a disseminação de Covid-ıg, e também que se confinem nas suas residências, conduzindo à depressão e prevalência de ideação suicida ${ }^{(13,14)}$.

A interrupção da continuidade dos tratamentos à dor crónica ou o seu tratamento inapropriado, conduz a efeitos no sistema imunitário, tornando o doente mais suscetível a contrair infeção por SARS-COV2 e assim padecer de Covid-19 ${ }^{(13)}$. Este fato relaciona-se com a ação da dor crónica sobre o sistema imunitário, na medida em que exerce múltiplos efeitos, incluindo imunossupressão em alguns doentes $^{(\mathrm{i} 4)}$. A terapêutica utilizada para manejo da dor crónica, conduz ela própria, a 
comprometimento do sistema imunitário como os opiáceos e os corticosteroides $^{(\mathrm{I} 4,15,16,17,23)}$.

Os doentes com dor crónica, que aguardam por avaliação e tratamento, vêem a sua condição degradar, sendo a recuperação espontânea rara ${ }^{(19)}$. Neste compasso de espera por avaliação da sua situação de dor, os doentes referem elevação da intensidade da mesma, o que traz consequências para a concretização das atividades de vida diárias, na medida em que interferem com a sua funcionalidade no dia-a$\mathrm{dia}^{(\mathrm{I} 4)}$, referindo-se que "doentes que esperam mais de 6 meses por avaliação, experienciam deterioração do seu estado de saúde e qualidade de vida, aumento dos níveis de dor e depressão"(18). Assim, conclui-se que não valorizar e tratar a dor crónica, se mostra mais prejudicial do que encetar um tratamento que se venha a revelar não ser o mais correto ${ }^{(18)}$.

São ainda identificados problemas gerais e específicos que ficaram exacerbados com o surgimento da pandemia por Covid-19 e que afetam os indivíduos com dor crónica. Neste sentido, os problemas de ordem geral identificados, prendem-se com abuso de opióides e o fracasso em educar e aconselhar os doentes. Já os problemas identificados de caráter específico, passam pelo aumento significativo da inatividade devido à imposição de confinamento para evitar a disseminação de infeção por SARS-COV2, bem como a necessidade de realizar quarentena ${ }^{(16)}$.

Em tempos de pandemia Covid-ı́, os doentes com dor crónica estão a ser negligenciados devido à longevidade da condição de dor, a necessidade de extensa avaliação diagnóstica com terapia multimodal e a necessidade de consultas presenciais para estabelecer uma relação de ajuda ${ }^{(26)}$. A impossibilidade de consultar um especialista em dor, leva a que os doentes com dor crónica procurem tratamentos alternativos e provavelmente inapropriados ${ }^{(27)}$.

Por tudo o que foi referido, o manejo da dor crónica não pode ser tido como condição não urgente em tempos de pandemia Covid-ı́ e o acompanhamento e seguimento dos doentes com dor crónica tem de ter continuidade.

- Soluções que promovam a continuidade do manejo da dor crónica em tempo de pandemia Covid-ı9 
Nas referências literárias consideradas, são apontadas soluções para que o acompanhamento e seguimento de doentes com dor crónica prossiga, de forma a que o manejo da dor ocorra, atendendo às medidas de segurança que a mitigação de disseminação do vírus SARS-COV̌ exige.

Como solução apontada, de forma geral pela maioria dos artigos considerados nesta SR, surge a telessaúde para continuar o seguimento de situações de dor crónica não urgente ou de longo termo ${ }^{(13,15,16,21-24)}$. Carateriza-se por comunicação interativa bidirecional em tempo real conduzida remotamente com um dispositivo audiovisual $^{\left({ }_{13}\right)}$, permite assistência ao doente de forma remota e permite a continuidade dos tratamentos e trabalhar estratégias de coping ${ }^{(18)}$.

As vantagens que a telessaúde oferece, passam por evitar exposição presencial desnecessária, redução da necessidade de uso de equipamentos de proteção individual e assim redução no consumo de recursos e melhorar o acesso aos cuidados de saúde, ao mesmo tempo que evita saturar as infraestruturas de saúde, já de si sobrecarregadas ${ }^{(15)}$.

Apesar das vantagens, são apontadas algumas limitações ao sistema de telessaúde, nomeadamente a questão da privacidade e proteção de dados, maior probabilidade de diagnósticos incorretos e impossibilidade de conduzir estudos imagiológicos de diagnóstico(22). A aplicação destes mecanismos informáticos a indivíduos com deficiência, idosos ou com comprometimento da cognição, pode ficar inviabilizado, o que também se constitui como limitação(15).

A evidência existente não mostra nenhuma diferença entre as terapias fornecidas por meios remotos face às terapias presenciais ${ }^{(18)}$. A introdução da telessaúde envolve conhecer as opções tecnológicas disponíveis e o treino dos profissionais de saúde para se adaptarem ao contexto remoto.

Além do acompanhamento remoto, através da telessaúde, fica também patente que a solução para dar continuidade ao manejo da dor cónica, passa por triar as situações de dor eletivas, urgentes e emergentes ${ }^{(14,21)}$, para identificar aquelas em que não é suscetível adiar ou cancelar procedimentos e consultas presenciais dado o risco de agravamento, intensificação da dor e declínio da funcionalidade ${ }^{(27)}$. Para proceder à triagem referida, é sugerido pela literatura considerada, que os profissionais de 
saúde envolvidos na tomada de decisão considerem os fatores de risco apresentados pelo doente e que o tornam mais suscetível a contrair infeção por SARS-COV2 (idade avançada, distúrbios psíquicos e sociais, patologia pulmonar e cardiovascular, patologia hepática e renal, imunossupressão, diabetes e obesidade), bem como pesar os benefícios/riscos da intervenção, para determinar a necessidade de intervenções clínicas presenciais ${ }^{(25,27)}$.

As referências consideradas, reportam também à criação de uma escala de acuidade para classificação da situação de dor do doente em eletiva, urgente ou emergente e assim determinar a necessidade de intervenções presenciais ou o seu adiamento $^{(27)}$ mas também por escassez de recursos e porque é “necessário um sistema ordenado para garantir que todos os doentes recebem tratamento adequado"(27). Uma vez estabelecido que as intervenções clínicas no âmbito da dor crónica têm de se realizar presencialmente pelo seu caráter urgente ou emergente, as referências consideradas, apontam para a necessidade de adotar medidas com vista a redução do risco de infeção por SARS-COV2. Desta forma, são definidas medidas gerais como a monitorização da temperatura dos doentes à entrada, da triagem de doentes com febre e sintomas respiratórios, da colocação de sinalética que reporte à necessidade de higiene das mãos, respeito pela etiqueta respiratória e distanciamento social, o uso de máscara cirúrgica pelos doentes e profissionais de saúde, sendo que, em caso de procedimentos que produzam aerossóis, os profissionais devem usar máscara com filtro de partículas, além de batas, luvas e viseiras ${ }^{(21)}$. A promoção de equipamento para lavagem/higienização das mãos, limitação de acompanhantes dos doentes ao número estritamente necessário e higienização correta das salas de observação/procedimento entre doentes estão também contempladas ${ }^{(21)}$.

Nesta sequência, tendo por base a literatura considerada, é ainda aconselhado a proceder-se a agendamentos das consultas e procedimentos, como forma de gerir o espaço, garantindo as medidas de distanciamento social adequadas, sugerindo-se a testagem dos doentes relativamente ao vírus cerca de $48 \mathrm{~h}$ antes da consulta ou procedimento, sendo o follow-up realizado de forma remota ${ }^{(24,25)}$.

Em suma, o momento atual de pandemia, deve constituir uma oportunidade de reorganização dos serviços da dor crónica, por forma a manter a sua continuidade. 
Novas formas de acompanhar os doentes com dor crónica têm de ser encontradas, por forma a que a gestão da dor continue a decorrer.

A pandemia por Covid-ı9 e o isolamento social que dela adveio, levou a reorganização das instituições de saúde, onde os serviços como os de gestão de dor foram relegados para segundo plano. Nesta sequência os doentes com dor crónica vivenciaram agravamento da dor e diminuição da qualidade de vida, como consequência da dificuldade em aceder a cuidados de saúde adequados.

Para dar continuidade ao acompanhamento de doentes com dor crónica a telessaúde constitui-se como solução, por possibilitar acompanhamento remoto.

Nos casos de dor, que pela sua complexidade não possibilitam adiar ou cancelar intervenções clínicas presenciais, é sugerido efetuar triagem, tomando por base os fatores de risco dos doentes e que os tornem mais suscetíveis para contrair SARSCov2, bem como desenvolver uma escala de acuidade que simplifique este processo de triagem.

O presente estudo apresenta limitações, dado a inexistência de estudos de evidência científica relevante, como estudos randomizados controlados e a inexistência de estudos atendendo às especificidades do contexto português. É ainda importante considerar que a pandemia por Covid-I9 se encontra em curso no momento da realização do presente estudo.

A presente SR poderá servir de base a estudos futuros. Neste sentido seria interessante realizar estudos sobre as consequências da pandemia por Covid-ı no manejo da dor crónica em Portugal, bem como um estudo que permitisse orientar a introdução de telessaúde no contexto português. Também se mostraria interessante encetar um estudo no sentido de desenvolver uma escala de acuidade, atendendo às características dos doentes com dor crónica em Portugal e que permitisse a categorização de situações de dor, para orientar para a resposta mais correta.

\section{REFERÊNCIAS}

Raja, S., Carr, D., Cohen, M., Finnerup, N., Flor, H., Gibson, S., Keefe, F., Mogil, J., Ringkamp, M., Sluka, K., Sung, X., Stevens, B., Sullivan, M., Tutelman, P., Ushida, T. \& Vader, K. (2020). The revised International Association for the Study of Pain definition of pain: concepts, challenges and compromises (traduzido por DeSantana, J. Perissinoti, D. Junior, J. Correia, L. Oliveira, C. \& Fonseca, P., 2020, Tradução para a 
língua portuguesa da definição revisada da dor pela Sociedade Brasileira para o Estudo da Dor, I-8). Consultado a I4 de dezembro de 2020. Recuperado em https://sbed.org.br/wp-content/uploads/2020/o8/Defini\%C3\%A 7\%C3\%A30-revisadade-dor_3.pdf;

Circular Normativa no o9/DGCG, de 14 de junho, da Direção Geral da Saúde (2003). A dor como $5^{\circ}$ sinal vital. Registo sistemático da intensidade da dor, p. 1-4. Consultado entre $I_{4} \mathrm{e}$ 2I de dezembro de 2020. Disponível em https://www.apeddor.org/documentos/DGS-dor_como_5_sinal_vital_-_2003.pdf;

Direção-Geral da Saúde (200I). Plano Nacional de Luta contra a Dor. Lisboa: DireçãoGeral da Saúde. ISBN: 972-9425-95-7;

Circular Normativa no II/DSCS/DPCD, de I8 de junho, da Direção Geral da Saúde (2008). Programa Nacional de Controlo da Dor, p. I-I6. Consultado entre I4 e 2i de dezembro de 2020. Disponível em https://www.dgs.pt/directrizes-da-dgs/normas-ecirculares-normativas/circular-normativa-n-IIdscsdpcd-de-I8062008-pdf.aspx;

Silva, S. (2018). Dor Crónica: a Doença, o Impacto e a Opiofobia (Trabalho final do Mestrado Integrado em Medicina, Faculdade de Medicina de Lisboa). Consultado entre I4 e 2i de dezembro de 2020. Disponível em http://hdl.handle.net/Io45I/42567;

Sapeta, P. (2007). Dor total vs Sofrimento: a interface com os Cuidados Paliativos. Revista Dor, I5(I): 16-2I. Consultado a I4 a 21 e dezembro de 2020. ISSN: 0872-48I4;

Oliveira, V., Ferreira, M., Morso, L., Albert, H., Refshauge, K. \& Ferreira, P. (2015). Patients' perceived level of social isolation affects the prognosis of low back pain. European Journal of Pain, 19(4), 538-545. DOI: 10.1002/ejp.578;

Johnson, K. \& Dunbar, R. (2016). Pain tolerance predicts human social network size. Scientific Reports 6, 25267. DOI: I0.1038/srep25267;

Mota, D., Ferreira, P. \& Leal, L. (2020). Produção científica sobre a COVID-ı9 no Brasil: uma revisão de escopo (pré-print). Scielo Preprints. Consultado entre I4 e 2I de dezembro de 2020. DOI: 10.1590/SciELOPreprints.464;

Peters M., Godfrey C., McInerney P., Munn Z., Tricco A., Khalil, H. (2020). Chapter II: Scoping Reviews. JBI Manual for Evidence Synthesis (2020 version), 406-45I. DOI: I0.46658/JBIMES-20-I2;

Moher, D., Liberati, A., Tetzlaff, J., Altman, D. \& The PRISMA Group (2009). Preferred Reporting Items for Systematic Reviews and Meta-Analyses: The PRISMA Statement. PLoS Medicine, 6(7): eroooo97, I-6. DOI:I0.1371/journal.pmedioooo97;

Piraccini, E., Byrne, H. \& Taddei, S. (2020). Chronic pain management in COVID-19 era. Journal of Clinical Anesthesia, 65, 109852. Consultado entre i4 de novembro e 2i de dezembro de 2020. DOI: 10.1016/j.jclinane.2020.109852; 
Puntillo, F., Giglio, M., Brienza, N., Viswanath, O., Urits, I., Kaye, A., Pergolizzi, J., Paladini, A. \& Varrassi, G. (2020). Impact of COVID-19 pandemic on chronic pain management: Looking for the best way to deliver care. Best Practice \& Research Clinical Anaesthesiology, 34(3), 529-537. Consultado entre I4 de novembro e 2I de dezembro de 2020. DOI: 10.1016/j.bpa.2020.07.0or;

Maan, M., Siddique, A., Iqbal, R. \& Ahmad, K. (2020). COVID-19 Pandemic and Chronic Pain Management: Impact and Suggestions. Pakistan Armed Forces Medical, 70(I), 413-417. Consultado entre I4 de novembro e 2I de dezembro de 2020. Disponível em https://www.pafmj.org/index.php/PAFMJ/article/view/4942/2790;

Ghai, B., Malhotra, N. \& Bajwa, S. (2020). Telemedicine for chronic pain management during COVID-I9 pandemic. Indian Journal of Anaesthesia, 64(6), 456-462. DOI: I0.4103/ija.IJA_652_20;

El-Tallawy, S., Nalamasu, R., Pergolizzi, J. \& Gharibo, C. (2020). Pain Management During the COVID-i9 Pandemic. Pain and Therapy, 9(2), 453-466. Consultado entre I4 de novembro e 2I de dezembro de 2020. DOI: doi.org/ro.1007/s40122-020-0oigo-4;

Shanthanna, H., Strand, N., Provenzano, D., Lobo, C., Eldabe, S., Bhatia, A., Wegener, J., Curtis, K., Cohen, S. \& Narouze, S. (2020). Caring for patients with pain during the COVID-19 pandemic: consensus recommendations from an international expert panel. Anaesthesia, 75(7), 935-944. Consultado entre I4 de novembro e 2i de dezembro de 2020. DOI: Io.IIII/anae.15076;

Eccleston, C., Blyth, F., Dear, B., Fisher, E., Keefe, F., Lynch, M., Palermo, T., Reid, M. \& Williams, A. (2020). Managing patients with chronic pain during the COVID-I9 outbreak: considerations for the rapid introduction of remotely supported (eHealth) pain management services. Pain Journal, I6I(5), 889-893. Consultado entre I4 de novembro e 2i de dezembro de 2020. DOI: 10.1097/j.pain.oooooooooooor885;

Li, L., Chew, A. \& Gunasekeran, D. (2020). Digital health for patients with chronic pain during the COVID-I9 pandemic. British Journal of Anaesthesia, 125(5), 657-66o. Consultado entre 14 de novembro e $2 \mathrm{I}$ de dezembro de 2020. DOI: Io.1016/j.bja.2020.08.003;

Clauw, D., Häuser, W., Cohen, S. \& Fitzcharles, M. (2020). Considering the potential for an increase in chronic pain after the COVID-I9 pandemic. Pain Journal, I6I(8), I6941697. Consultado entre i4 de novembro e 2I de dezembro de 2020. DOI: https://doi.org/Io.1097/j.pain.oooooooooooor95o;

Cohen, S., Baber, Z., Buvanendran, A., McLean, B., Chen, Y., Michael Hooten, W., Laker, S., Wasan, A., Kennedy, D., Sandbrink, F., King, A., Fowler, I., Stojanovic, M., Hayek, S. \& Phillips, C. (2020). Pain management best practices from multispecialty organizations during the COVID-I9 pandemic and public health crises. Pain Medicine, 2I(7), I33I-1346. Consultado entre i4 de novembro e 2I de dezembro de 2020. DOI: doi.org/Io.1093/pm/pnaai27; 
Montero, A. \& Vasconcelos, S. (2020). COVID-19 y dolor crónico: muchos interrogantes y pocas certezas. Revista Semergen, 46(6), 365-367. Consultado entre I4 e 2I de dezembro de 2020. DOI: I0.1016/j.semerg.2020.08.002;

Coluzzi, F., Marinangeli, F. \& Pergolizzi, J. (2020). Managing chronic pain patients at the time of Covid-19 pandemic. Minerva Anestesiologica, 86(8), 797-799. Consultado entre I4 de novembro e 2I de dezembro de 2020. DOI: doi.org/I0.23736/s03759393.20.14666-2;

Hascalovici, J. \& Shaparin, N. (2020). The other side of the Covid-r9 curve: A model for the safe reintegration of elective interventional pain procedures. Pain Physician, $23(4$ Special Issue), 449-45I. Consultado entre i4 de novembro e 2i de dezembro de 2020 . PMID: 32942800;

Shah, S., Diwan, S., Soin, A., Rajput, K., Mahajan, A., Manchikanti, L., Kaye, A., Hirsch, J. \& Gharibo, C. (2020). Evidence-Based Risk Mitigation and Stratification During COVID-ig for Return to Interventional Pain Practice: American Society of Interventional Pain Physicians (ASIPP) Guidelines. Pain physician, 23(4S), 16I-182. Consultado entre i4 de novembro e 2i de dezembro de 2020. PMID: 32942784;

Bara, G., Ridder, D., Vatter, H., \& Maciaczyk, J. (2020). Between scylla and charybdis: Navigating chronic pain patients through the Covid-I9 and the opioid pandemic. Pain Physician, $23(4 \mathrm{~S}), 469-47 \mathrm{I}$. Consultado entre I4 de novembro e 21 de dezembro de 2020. PMID: 32942809;

Gharibo, C., Sharma, A., Soin, A., Shah, S., Diwan, S., Buenaventura, R., Nampiaparampil, D., Aydin, S., Bakshi, S., Abdi, S., Jha, S., Cordner, H., Kaye, A., Abd-Elsayed, A., Candido, K., Knezevic, N., Atluri, S., Wargo, B., Sanapati, M., Datta, S., Hirsch, J., Rajput, K. \& Manchikanti, L. (2020). Triaging Interventional Pain Procedures During COVID-I9 or Related Elective Surgery Restrictions: EvidenceInformed Guidance from the American Society of Interventional Pain Physicians (ASIPP). Pain Physician, 23(4), 183-204. Consultado entre 14 de novembro e 21 de dezembro de 2020. PMID: 32942785 . 